\title{
Evolution of Seal Cutting Symbol Elements
}

\author{
Bo Peng \\ Zhuhai College of Jilin University \\ Zhuhai, China
}

\begin{abstract}
Seal cutting is a traditional art and it is the predecessor of one of the four inventions-printing which closely combined with the calligraphy, so far it has 3000 years of history. Also it is the prototype of Chinese logo design so its cultural status can not be ignored. Its origin, development, prosperity is inseparable from its time, and it is closely related to the political, economic and cultural environment of that time. With the continuous improvement of aesthetic requirements, changes of seal cutting symbols are also related with other art-related content, such as epigraphy, philology, literature, painting and so on. The printed text mostly is seal script so it is called seal cutting. Because seal symbol has obvious characteristics, mostly use pictographic elements, its form mostly is elliptic, its font has great symmetry, smooth and neat, so seal cutting use seal script until now. Due to the impact of calligraphy elements, the appreciation of seal cutting is mainly from "shape", "meaning" and "lingering charm" three aspects. We need to watch their shape, understand its meaning, pass on its lingering charm. Seal cutting emerged in Qin Dynasty and began to develop since then; Wei and Jin Dynasties is the degradation of decay; Sui ,Tang and Song Dynasties is the fission and incubation period; Yuan and the mid-Ming Dynasty is the turning and awaken period; the late Ming to the Qing Dynasty is the prosperous, peak period. In different periods, the aesthetic elements constant evolution, however, the functionality is not change so much, it always be the symbol of personal identity. But with the deduction of aesthetic symbol, seal cutting art as a traditional symbolic elements, through purposeful refining, applied to various fields, such as the name seal with simplify font, or description seal used to proof things, or title seal based on different content in paintings ; also extracted its traditional symbol style and applied to the design of modern logo field and so on. Therefore, this article will analyze the communicate procedure of symbolic elements from its process of evolution.
\end{abstract}

Keywords—seal cutting; element; symbol; semantics

\section{The DeVelopment Process of Chinese SeAL CUTTING}

The symbolic value of Chinese seal cutting art is from many ways and reflected from multi-level with its own development track.

Qin seal is the first peak period of Chinese seal cutting art history. The main sign is Qin unified the world, forming a unified system. In Qin dynasty not only developed a relatively complete system of official seal, and the seal text is not like the complicated situation in the Warring States period, they unified the text symbols of "copy printed seal" (Xiaozhuan), they set up regulatory requirements for the text to make it symmetry. It has a huge impact on future generations. Han dynasty inherited Qin's system, made further improvement for the system. Second is it formed its own style. Especially the Han seal is flat straight and square with dignified deportment and balanced harmonious and natural cutting technology. It fit for the ancient Philosophy of the unity of man nature. It makes the Han seal style can be identified at a single glance. Third, the technology is mature. Qin and Han seal (Qin's short, mainly Han seal) have shown remarkable skill and exquisite craft no matter cast or chisel. Even the "rushed seal", engraved with the graver directly on copper, skill practices are very skillful mastery.

Seal cutting in Wei-Jin Southern and Northern Dynasties is in recession, it did not inherit the characteristics of Han text symbols, and its production is rough and with no charm. The Qin and Han seal element has not been inherited and developed.

Ming Qiong Seal is the second peak period of the Chinese seal cutting art history. First, the stone material is widely adopted; Second is the popularity of"Yin Pu"; Third is different genres appears. The most important person in this period are He Zhang, He Lv, they pass the essence of Qin Han seal, they "use text to explain knowledge, correct the mistakes from ancient seal script; use exquisite writing technology to improve the weak of Jiudiewen ; use masterly Han seal technology to improve the shallow and crude old technology; use Qingtian stone as seal material which suitable for Qin cutting knife to replace the copper and rhinoceros teeth seal material; use exquisite side inscription expansion to enrich the seal art world. In the Qing Dynasty, the rise of Jianand Hui School, and Deng Shiru, Wu Xizai , Zhao Zhijian, Huang Shiling, Wu Changshuo and other masters have emerged. Therefore, during this period, rich seal cutting style has left a cultural platform for future generations about marked symbol art.

Chinese literati seal cutting has entered its heyday in midQing Dynasty. Jian and Hui factions developed broadly and appeared many famous masters. Until the Four Masters in the late Qing period, it is the pinnacle. At the beginning of the Republic period, the $\mathrm{Wu}$ Changshuo who is excellent in both skills and moral integrity has dominated the seal field for decades. Thereafter, although it never appears seal cutting masters who can compare with the late Qing Dynasty Four Masters but with the strong influence of the four masters, the Republic period seal cutting filed still showing abnormal prosperity and full of artists gathered. It provides a worship peak for future generations of seal cutting designs. 
In Republic Period, the style of seal cutting shows diversified development trend. Since the progress of the times and strengthen of innovation sense, most engraving people can pursuit style variation.Although the Hai Pai engraving person consider $\mathrm{Wu}$ Changshuo as master, Yue Pai engraving people affect the influence of Huang $\mathrm{MuFu}$, Zhejiang engraving people called them-self as new Zhej Pai, But they do not only stick to the old technology or picked up the pace in the form of simulation. Within each faction, they influence each other and many engraving people learned from many factions with complex style changes, it is more colorful than any other time in history. Spread and impact of seal faction, already without geographical limitations, many engraving person is difficult to simply belong to a particular faction. The precise style represented by Zhao Shifeng and wild style represented by Qi Baishi, both achieve the ultimate in the precise and powerful work in seal cutting, they opened up a new realm for Modern seal cutting art. Especially with large number of unearthed Yin $\mathrm{Xu}$ bones, ancient seals in Warring States, and Qin and Han lute and deep research about them, the reference range of creative seal cutting has been greatly expand. The application for the engraving people to ancient texts shows the trend of fine study into expert. Engrave bone text into seal, smelt and innovate the ancient seal with Jin Wen from Shang Zhou Dynasty, enrich and modify Qin Han seal script with Qin Han inscriptions on ancient bronzes and stone tablets, these new creative technology has been learned and used by more and more engraving people and it is the older engraving people from Ming and Qing Dynasties can not dream. In this period it appears communities such as West seal community, to promote the communication and exchange of seal cutting and make contribution for the development of modern and contemporary art of seal cutting.

\section{IMPORTANT TURNING POINT OF THE DEVELOPMENT OF ChInESE SEAL CUTTING}

Another turning point is the Yuan Dynasty. After Qin and Han dynasty, seal cutting art has a silent period until the Yuan Dynasty it appeared vigorous signs of life. The main flag person is Zhao Mengfu who add Yuan Zhu font into seal cutting. He is a prominent calligrapher, painter, poet, although he did not direct cut the seal, but often personally design seal draft for cutting. He uses elegantly Yu Jin seal to correct Jiu die seal. The style of writing is rich and vivid, handsome and elegant, which make a great influence to the seal cutting history. Second is the signature seal prevailed, because Mongolians who became the master of the Central Plains do not know a lot of Chinese characters, so they use signatures to replace seal which has a unique, natural-looking. This gives us a revelation that art is from the folk, although Yuan Dynasty is short, but it directly opens an era, and laid a good foundation for the Ming and Qing seal cutting prosperous. So we called the big turning point.

In Chinese seal cutting art history, people always have this question: Why the two peak period of Chinese seal cutting art is about a thousand years apart? Some people believe that Qin and Han is just a natural interest in obtaining the childish, only Ming and Qing seal cutting is the result of consciously art pursuing. Was it true? After careful combing, analysis and research, we believe the artistic achievement of Qin and Han seal cutting is not like that, it has a long history. A large number of unearthed ancient seal from Warring States period explained this point. Before the Warring State period, there must be a certain evolution period. Bronze inscriptions from West Zhou Dynasty are so beautiful, so his sister art from seal cutting must also be relatively mature, only need evidence to prove. Han seal still carries Han style into the late Eastern Han Dynasty until the Wei and Jin dynasty, but gradual enter the end. Following Sui, Tang and Song three dynasties, the seal cutting art fission occurs, on one hand government control seal gradually into a dead end, on the other hand in the birth of the private seal cutting. Ding Jing from Xileng Bajia said: "The ancient seal cutting always has innovative ideas and wishes to make it unique and remarkable like the clouds on the mountain. The six dynasties' seal cutting also very fine and ingenious, and it does not follow the ancient Qin Han seal technology."The fission in Tang and Song dynasty was foreshadowing of the turning point in Yuan Dynasty. This turning is completed until mid-Ming dynasty.

According to the seals spread from the Warring States to Ming Qing, the different Chinese fonts (big Seal, small Seal Script, Qin copy printed seal, Han Miao seal, birds and insects book, official script, regular script, Jiu die seal since the Tang and Song, song words etc.) are all used on seal. Due to some fonts is not easy to form a rich and varied formal beauty on the seal stone, so the major font for seal cutting is still ancient text, Miao seal and small seal script. With the evolution of the text, in different periods the characteristics of seal script are quite different. Ancient engraving people are familiar with the popular seal script at that time and cleverly use its characteristics to create many perfect works, so to form the unique artistic seal cutting style of various ages.

Symbolic elements discussion not only based on dynasties change, we must directly explore the nature of seal cutting art elements. One element is the font, copy printed seal method is sublime and change freely. A seal word can be long or short, narrow or broad ,square or round, fat or thin, increase or reduced; in the small sized seal surface, whether 2-3 words, 45 words or even more than a dozen words, dozens of words, can be mixed together cleverly. This is what other fonts can not do. Second, element is art, literati exploring seal cutting material, while also exploring seal colors. After Tang dynasty, seal do not have lute, and it used "Paper" element, so it must has color. At first it uses ink color, later usually use red color, and occasionally use Te and Yin color. In Song dynasty, it uses honey for the color and in Yuan dynasty it uses water to make the color, in Ming dynasty it start to uses oil ink for seal. Different color used in different places, especially the "red" color highlights the unique artistic charm of the seal cutting. The use of different colors conveys its unique charm depending on the different symbol elements. Third element is function, the application is also expanding. Private seal has been expanded on the basis of official seal. Such as the "reign title seal", Tang Taizong crates "Zhen guan": two words together and Tang Xuanzong's "Kai yuan" seal, not only start the reign title seal but also start the collection seal of paintings appreciation. Until now there are a lot of calligrapher and painters left "eighty's", "ninety's", "late seventy's" seal on 
their works. Connoisseur seal is mainly used in calligraphy, paintings and book collection for appreciation. Song Huizong, Song Gaozong the two emperors of Song dynasty also use seals on calligraphy and paintings a lot. Emperors in Qing dynasty is even more, emperor Kangxi and Qianlong collections are a lot. For those emperors, treasure in the country is the treasure their home, home is the country, so there is no difference between official seal and private seal. This has a high value of cultural value and artistic value. Fourth, element is seal skill, cutting skill and carving skill. Seal skill can not be simply interpreted as "copy the text ". Seal script is mature text, in line with "six meanings" and with strong regularity, so it is selected as the seal font .Of course, with the development of text, there are changes for seal text, but generally the pattern is unchanged. So seal skill is actually seal script skill. Sealed text word first should be correct, sound accurate is not scientific and credible; secondly it should be unity and harmony, later is about beauty and artistry. The socalled "cutting skill ", is the overall layout of the seal and the layout method. Knife skill is the skill to use the cutting knife," Hand should connect with heart so it can work together perfectly." The ancient people attached great importance to the knife skill, they believe it is the most difficult part, cutting skill is the middle difficult and seal skill is the last. Seal and cutting skill can learn to be perfect, but only knife skill can not be learn to perfect. The seal cutting knife skill art is divided into the Chong dao and qie dao two type. Chongdao uses knife smooth and clean without modification, the carved line does not require thickness symmetry, but for the vigorous and upright; Qiedao uses knife slowly with repeated small cuts, carved lines have thickness, irregular, undulating and other changes, with a strong sense of rhythm.

\section{CONCLUSION}

According to the seals spread from the Warring States to Ming Qing, the different Chinese fonts (big Seal, small Seal Script, Qin copy printed seal, Han Miao seal, birds and insects book, official script, regular script, Jiu die seal since the Tang and Song, song words etc.) are all used on seal. Different font has its own unique aesthetic features, but after unified text, ancient seal usually use ancient text, Miao seal and small seal script which are, easy to identify and easy to simplify. Such as the Beijing Olympics logo design, it inherits the traditional art form of seal cutting to show the status of Chinese culture to the world through this symbol. With the ever-expanding range of applications, text symbols in new era aesthetic rules, change the contents of a rich form, this form of application reflects the deep historic charm of traditional Chinese culture. And while deeply study of seal cutting art, expending into the other fields of traditional art, bring the essence of traditional culture into design thinking, so to flexible use symbol elements. The traditional culture of symbolic elements would convey China's rich artistic content, I believe this design will show its unique charm in the international design.

\section{REFERENCES}

[1] "Chinese seal cutting techniques book" Li Gangtian Henan Fine Arts Publishing Company.
[2] First Edition 2008.04

[3] "Logo art"Lu Yaoshi China Peace Publishing Company 1999.09.

[4] "logo design"Huang Kai, Zhai Yun,Gao Yuan Hefei University og Technology Press Company 2005.08.

[5] "Seal cutting art" Wu Huichen, Wu Jianying Tianjin People's Fine Arts Publishing Company 2007.01. 